\title{
ПРИМЕНЕНИЕ ЭЛАСТОМЕРОВ, МОДИФИЦИРОВАННЫХ УГЛЕРОДНЫМИ НАНОТРУБКАМИ ПРИ СОЗДАНИИ САМОРЕГУЛИРУЕМЫХ ЭЛЕКТРОНАГРЕВАТЕЛЕЙ И МАТЕРИАЛОВ ДЛЯ ЗАЩИТЫ ОТ ЭЛЕКТРОМАГНИТНОГО ИЗЛУЧЕНИЯ
}

\author{
А.В. Щегольков, А. В. Щегольков, Ф.Ф. Комаров, И. Д. Парфимович \\ АЛЕКСАНДР ВИКТОРОВИЧ ЩЕГОЛЬКОВ - к.т.н, доиент кафедры «Техника и технологии производства \\ нанопродуктов», ФГБОУВО ТГТУ.E-mail: energynano@yandex.ru.
АЛЕКСЕЙ ВИКТОРОВИЧ ЩЕГОЛЬКОВ - аспирант кафедры «Техника и технологии производства нанопродуктов», ФГБОУ ВО ТГТУ.E-mail: alexxх5000@mail.ru. \\ ФАДЕЙ ФАДЕЕВИЧ КОМАРОВ - д.ф.-.м.н., заведующиий лабораторией НИИПФП им. А.Н. Севченко БГУ. \\ E-mail: komarovF@bsu.by.
}

ИВАН ДМИТРИЕВИЧ ПАРФИМОВИЧ - аспирант Белорусского государственного университета. E-mail: irongrivus71@gmail.com.

392000, г. Тамбов, ул. Советская, д. 106. Тамбовский государственный технический университет (ФГБОУ BO TГТУ).

220045 г. Минск, Республика Беларусь, ул. Курчатова 7. НИИПФП им. А.Н. Севченко БГУ.

В статье представлены результаты исследований эластомеров модифицированных многослойными углеродныли нанотрубками (МУНТ). В качестве полимерной основы был использован эластомер - полидиметилсилоксан, а наполнителем являлись МУНТ, синтезированные на $\mathrm{Co}-\mathrm{Mo} / \mathrm{Al}_{2} \mathrm{O}_{3}-\mathrm{MgO} \mathrm{u} \mathrm{Fe-Co/}_{2.1} \mathrm{Al}_{2} \mathrm{O}_{3}$ катализаторах.

В ходе исследований, был проведен структурный анализ размеров частии МУНТ, в результате чего было установлено, что МУНТ синтезированные на Со-Мo/Al $\mathrm{O}_{3}-\mathrm{MgO}$ катализаторе имеют наружный диаметр около 25-35 нм и внутренний диаметр 10-15 нм, а MУНT на Fe-Co/ ${ }_{2.1} \mathrm{Al}_{2} \mathrm{O}_{3}$ имеют наружный диаметр 5-15 нм и внутренний диаметр 5-7 нм. Изучена электрическая проводимость полученных нагревательных элементов, максимальная электропроводность $\left(1,66 \cdot 10^{-1} \mathrm{CM}^{\circ} \mathrm{cm}^{-1}\right)$ наблюдалась у композита, изготовленного на основе полидиметилсилоксан, модифицированного 7 масс.\% $\mathrm{MYHT} \mathrm{на} \mathrm{Co-Mo/Al} \mathrm{O}_{3}-\mathrm{MgO}$ катализаторе, а максимальная интенсивность тепловыделения, в сочетании с равномерным распределением температурного поля, соответствовала образиу с МУНT на $\mathrm{Fe}$-Co/ ${ }_{2.1} \mathrm{Al}_{2} \mathrm{O}_{3}$ катализаторе, при этом максимальная температура нагрева $102{ }^{\circ} \mathrm{C}$, соответствовала постоянному напряжению $18-24$ В.

Исследования процессов взаимодействия электромагнитного излучения (ЭМИ) с изготовленными образиами на основе МУНТ синтезированных на катализаторах $\mathrm{Co}-\mathrm{Mo} / \mathrm{Al}_{2} \mathrm{O}_{3}-\mathrm{MgO}$ и $\mathrm{Fe}-\mathrm{Co} /{ }_{2.1} \mathrm{Al}_{2} \mathrm{O}_{3}$ noказали, что образиы нагревательных элементов выполненные с $\mathrm{MYHT} \mathrm{на} \mathrm{Fe-Co/}{ }_{2.1} \mathrm{Al}_{2} \mathrm{O}_{3}$ катализаторе демонстрирует широкополосное ослабление (до 20 \%) падающего излучения, в то же время, нагревательные элементы полученные на основе МУНТ синтезированных на Со-Мо/Al $\mathrm{O}_{3}-\mathrm{MgO}$ катализаторе проявляют способность к экранированию электромагнитного излучения в диапазоне от 26 до 40 ГГц на уровне $15 \%$.

Ключевые слова: многослойные углеродные нанотрубки (МУНТ), полидиметилсилоксан, нагревательный элемент. 


\title{
THE USE OF ELASTOMERS MODIFIED WITH CARBON NANOTUBES WHEN CREATING SELF-REGULATING ELECTRIC HEATERS AND MATERIALS FOR PROTECTION AGAINST ELECTROMAGNETIC RADIATION
}

\author{
A.V. Shchegolkov ${ }^{1}$ A. V. Shchegolkov ${ }^{1}$, F. F. Komarov ${ }^{2}$, I. D. Parfimovich ${ }^{2}$ \\ ${ }^{1} 106$, Sovetskaya Str., Tambov, 392000, Russia. Tambov State Technical University. \\ 27 Kurchatova str., Minsk, 220045, Republic of Belarus. A.N. Sevchenko Institute of Applied Physical Problems \\ of Belarusian State University.
}

In the article the study's results of elastomers modified by multilayer carbon nanotubes (MCNTs) are presented. An elastomer, polydimethylsiloxane, was utilized as the polymer base while MCNTs synthesized on Co-Mo/ $\mathrm{Al}_{2} \mathrm{O}_{3}-\mathrm{MgO}$ and $\mathrm{Fe}-\mathrm{Co} /{ }_{2.1} \mathrm{Al}_{2} \mathrm{O}_{3}$ catalysts were employed as an additive.

The structural analysis of MCNT particles sizes was carried out. It showed that MCNTs synthesized on Co-Mo/ $\mathrm{Al}_{2} \mathrm{O}_{3}-\mathrm{MgO}$ catalysts have outer diameter of 25-35 $\mathrm{nm}$ and inner diameter of 10-15 $\mathrm{nm}$, while MCNTs synthesized on $\mathrm{Fe}-\mathrm{Co} /{ }_{2.1} \mathrm{Al}_{2} \mathrm{O}_{3}$ catalysts have outer diameter of 5-15 $\mathrm{nm}$ and inner diameter of 5-7 $\mathrm{nm}$.

The electrical conductivity of the heating elements samples was studied. The maximum electrical conductivity $\left(1,66 \cdot 10^{-1} \mathrm{Cm} \cdot \mathrm{cm}^{-1}\right)$ was observed for a composite based on polydimethylsiloxane modified with $7 \mathrm{wt} \% \mathrm{MCNTS}$ (synthesized on the Co-Mo/Al $\mathrm{O}_{3}-\mathrm{MgO}$ catalyst) while the sample modified with MCNTs (synthesized on the Fe$\mathrm{Co} /{ }_{2.1} \mathrm{Al}_{2} \mathrm{O}_{3}$ catalyst) had the maximum heat release rate coupled with evenly distributed heat flow pattern. The maximum heating temperature of $102{ }^{\circ} \mathrm{C}$ corresponded to a constant voltage of $18-24 \mathrm{~V}$.

The studies of electromagnetic radiation interaction with the samples were executed. In those studies, heating element samples made of MCNTs (synthesized on the $\mathrm{Fe}-\mathrm{Co} /{ }_{2 . \mathrm{I}} \mathrm{Al}_{2} \mathrm{O}_{3}$ catalyst) demonstrated wide-band attenuation of impinging radiation (up to $20 \%$ ) while heating element samples made of MCNTs (synthesized on the Co-Mo/ $\mathrm{Al}_{2} \mathrm{O}_{3}-\mathrm{MgO}$ catalyst) showed the ability of electromagnetic shielding in the range of 26 to $40 \mathrm{GHz}$ at the level of $15 \%$.

Keywords: multilayer carbon nanotubes (MCNT), polydimethylsiloxane, heater element.

\section{Введение}

В современном материаловедении, важное значение имеют исследования и разработки новых МУНТ - способных улучшить функциональные свойства различных полимеров. Новым этапом развития технологий электронагрева, стало появление полимерных композитов с электропроводящими углеродными наполнителями [1-3]: электропроводящая сажа, уголь, графен, фуллерены и др. $[4,5]$. Одним из самых востребованных и изученных углеродных материалов для повышения электрофизических свойств полимерных композитов является электропроводящая сажа [6-8], но с удешевлением технологии синтеза углеродных нанотрубок (УНТ) и МУНТ различной структурной конфигурации, которые по ряду характеристик существенно превосходят другие углеродные мате- риалы, становится актуальной задачей их получение и применение в качестве наполнителя. Коммерческая привлекательность УНТ и МУНТ, стала возможна благодаря большей насыпной плотности, высокой площади удельной поверхности, а также за счет оптимальных тепло - электрофизических параметров $[9,10]$.

Эффект саморегулирования электронагревательных элементов, возможен в ряде случаев за счет положительного температурного коэффициента сопротивления и может возникать по нескольким причинам [11]. Одними из основных являются: наличие фазового перехода в полимере, происходящего до температуры плавления полимера и разницы коэффициентов теплового расширения между полимерной матрицей, и проводящими наполнителями при смене температурных условий окружающей среды. Также данный эффект 
возможен благодаря следующим факторам: химическому составу наполнителя и полимерной матрице, размеру частиц наполнителя, массовому содержанию электропроводящей фазы, взаимодействию между частицами наполнителя и полимерной матрицы и др. [12].

Электронагревательные элементы на основе полимерной композиции могут различаться как составом полимеров, так и электропроводящими компонентами, в качестве полимерной основы используют следующие материалы [13-15]: эластомеры, полиэтилен низкого давления (ПНД), полилактид (ПЛА), сополимер этилена с винилацетатом (ЭВА) и др. Основными проблемам и встречающимися при изготовлении электронагревательных материалов на полимерной основе, является выбор оптимальной концентрации углеродного наполнителя, а также технологии введения и его однородного распределения в объеме полимерной матрицы. Обычно электронагревательные материала изготавливают по растворной технологии, внесением электропроводящей добавки в расплав, полимеризацией in situ [16-18].

Для обеспечения электромагнитной совместимости отдельных блоков высокочастотной радиоаппаратуры, используют композиционные материалы на основе полимерных композиций с УНТ, данные радиопоглощающие материалы, активно взаимодействуют с электромагнитным излучением и обладают рядом преимуществ перед изделиями из сплошных магнитных или диэлектрических материалов. Многослойные широкополосные поглотители электромагнитного излучения на основе композиционных материалов используют принцип преобразования электромагнитной энергии в тепло [19].

Разработка радиопоглощающих материалов с заданным коэффициентом затухания электромагнитного излучения имеет большое значение в различных технических областях [20]. При этом, данные материалы должны обеспечивать необходимое ослабление отражательной способности электромагнитных волн ближнего или дальнего поля для различных объектов, с различными углами падения и поляризации. Радиопоглащающие материалы должны иметь небольшую массу, низкую стоимость, хорошие термические свойства, механические и химические свойства [21]. В качестве наполнителей композитов на данный момент активно используют наноматериалы, физические свойства которых существенно отличаются от массивных образцов этих же материалов: наноструктурные магнитные материалы [22] и углеродные наноразмерные структуры, в частности УНТ [23].

Получение эффекта температурного саморегулирования в полимерных электронагреватель- ных элементах модифицированных УНТ и МУНТ, а также преобразование ЭМИ в тепловую энергию в полимерных композиция, являются перспективными задачами. Исходя из этого, были сформулированы следующие задачи:

1. Исследовать морфологию, структуру, а также размерные параметры МУНТ, полученных на разных катализаторах $\left(\mathrm{Co}-\mathrm{Mo} / \mathrm{Al}_{2} \mathrm{O}_{3}-\mathrm{MgO}\right.$ и $\mathrm{Fe}-\mathrm{Co} /{ }_{2.1} \mathrm{Al}_{2} \mathrm{O}_{3}$ ).

2. Изучить электропроводность эластомера полидиметилсилоксана, модифицированного МУНТ.

3. Установить влияние различных концентраций МУНТ в эластомерах, на процесс тепловыделения при заданном напряжении.

4. Исследовать модифицированные материалы на отражение, прохождение и поглощение электромагнитного излучения.

\section{Материалы и методы}

В качестве модифицирующих углеродных материалов были использованы МУНТ, полученные по CVD-технологии на Co-Mo/ $\mathrm{Al}_{2} \mathrm{O}_{3}-\mathrm{MgO}$ (МУНT-1) и $\mathrm{Fe}-\mathrm{Co} / 2.1 \mathrm{Al}_{2} \mathrm{O}_{3}$ (МУНТ-2) катализаторах. В основе полимерной матрицы был использован эластомерполидиметилсилоксан компаунд «Силагерм 8030» (ООО «ЭЛЕМЕНТ 14», Москва, Россия). Удельную площадь поверхности МУНТ измерили при помощи анализатора «ASiQwin» («QUANTACHROME Instruments», Флорида, США). Морфологию поверхности МУНТ исследовали с помощью сканирующего электронного микроскопа «Hitachi H-800» (Япония). Спектры комбинационного рассеяния (КР) модифицированных эластомеров были измерены на Романовском микроскопе DXR Raman Microscope (Thermo Scientific, США) при длине волны возбуждающего лазера 532 нм. Удельное объемное и поверхностное электрическое сопротивление измеряли с помощью мультимметра «UNI-TUT61B» (Китай) с пределом измерения электрического сопротивления до 60 МОм.

Исследования процессов тепловыделения на поверхности нагревательного элемента производили при помощи тепловизора Testo 875 (Германия, Testo). Нагревательные элементы, на основе полидиметилсилоксана, модифицированные МУНТ-1 и МУНТ-2 обозначали в соответствии с типом модификатора и его массовым содержанием - КУНТ $-1.1 \ldots 1.7$ и КУНТ $-2.1 \ldots 2.7$ соответственно, где $1 . . .7$ масс.\% МУНТ.

Для определения взаимодействия ЭМИ в СВЧдиапазоне с композитными материалами (прохождение, отражение и поглощение ЭМИ) использовался скалярный анализатор цепей, построенный на основе генератора качающейся частоты, пря- 
моугольного волноводного измерительного тракта, блока анализатора и системы обработки сигналов. Измерения проводились в четырех диапазонах частот: 8-12 ГГц, 12-18 ГГц, 18-26 ГГц, 2640 ГГц. Исследуемые образцы композитов вырезались в форме параллелепипеда с размерами в $23 \times 10 \times 2$ мм, $16 \times 8 \times 2$ мм, $11 \times 5,5 \times 2$ мм, 7,2×3,4×2 мм. Размер исследуемых композитных материалов жестко связан с размерами канала волновода, который определяет частотный диапазон измерений.

\section{Результаты и их обсуждение}

МУНТ, синтезированные на различных катализаторах отличались друг от друга морфологией, что следует из данных, представленных на рис. 1. В обоих случаях наблюдаются нитевидные образования, состоящие из нескольких графеновых слоев, длиной около 2 мкм и более, которые объединены в пучки.

Проведенные исследования показали, что удельная поверхность для МУНТ-1 и МУНТ-2 составляла 347 мл/г и 276 мл/г соответственно. Согласно проведенных исследований МУНТ-1 имеют наружный диаметр около 25-35 нм и внутренний диаметр 10-15 нм. МУНТ-2 имеют наружный диаметр 5-15 нм и внутренний диаметр 5-7 нм.

На основании анализа КР спектра МУНТ, толщина стенки МУНТ-1 составляла 8,75 нм, что в 4,38 раза больше значения толщины стенки МУНТ-2 (4 нм). В результате синтеза МУНТ-1 в отличие от МУНТ-2 обладает большим количеством графеновых слоев, из которых они образованы. Из этого следует, что масса МУНТ-1 больше массы МУНТ-2 при том, что оба материала обладают близким значением занимаемого объема. При этом насыпная плотность для МУНТ-1 и МУНТ-2

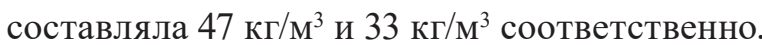

По результатам лазерного анализа размеров частиц образца МУНТ-1 получены следующие данные, в соответствии с которыми размеры ча- стиц колеблются от 1 до 100 мкм, основная весовая фракция частиц имеет размеры 20-54 мкм. Частицы размером до 54,3 мкм составляют 91,4 \% всей массы образца МУНТ-1.

По данным лазерного анализа размеров частиц МУНТ-2, их размеры колеблются от 1 до 220 мкм, при этом основная весовая фракция частиц имеет размеры 30-80 мкм 98,3 \% весовой доли образца приходится на частицы размерами до 121 мкм.

МУНТ-1 является более монодисперсным и состоит из более мелких частиц в сравнении с МУНТ-2. Мелкие частицы, присутствующие в МУНТ-1, являются их агрегатами, а не индивидуальными углеродными нанотрубками. Катализатор (Co-Mo/ $\mathrm{Al}_{2} \mathrm{O}_{3}-\mathrm{MgO}$ ), который использован в синтезе МУНТ-1 позволяет получить более однородные по размеру агрегаты с меньшим диаметром. Исследования, проведенные методом динамического рассеяния света, показали, что диаметр частиц МУНТ-1 и МУНТ-2 и их распределение практически одинаково. В МУНТ-1 и МУНТ-2 присутствуют частицы различных размеров в диапазоне от 200 нм до 24 мкм. С наибольшей интенсивностью в МУНТ-1 и МУНТ-2 детектируются частицы диаметром 1,4-1,6 мкм. Средний диаметр частиц в МУНТ-1 и МУНТ-2 составлял 2,48 мкм и 2,44 мкм соответственно.

Структурные особенности МУНТ-1 и МУНТ-2 повлияли на электропроводность нагревательного элемента на основе КУНТ -1.1...1.7 - КУНТ -2.1... 2.7 (рис. 2). Исходя из результатов измерений электропроводности следует, что максимальная электропроводность характерна для КУНТ -1.1 и КУНТ -1.7-0.1 и 0,16 См·см ${ }^{-1}$ соответственно. Меньшей электропроводностью 0,02 и 0,068 $\mathrm{CM}^{\cdot} \mathrm{cm}^{-1}$ обладают КУНТ-2.1 и КУНТ-2.7.

Температура нагрева образцов (рис. 3), изготовленных из полидиметилсилоксана с различным содержание масс. \% МУНТ-1, варьировалась от 32,9 до $102{ }^{\circ} \mathrm{C}$ (рис. 3a, 3b и 3c), при этом удельная площадь поверхности составляла $8,7 \mathrm{~cm}^{2}$.

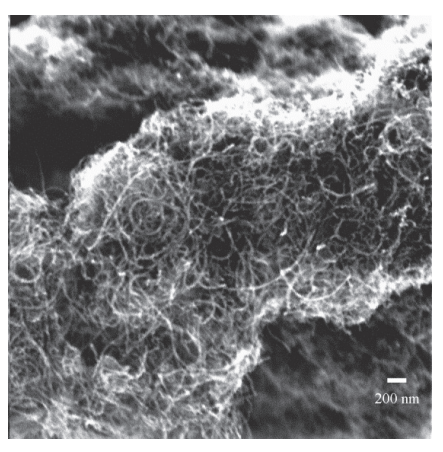

$\mathbf{a}$

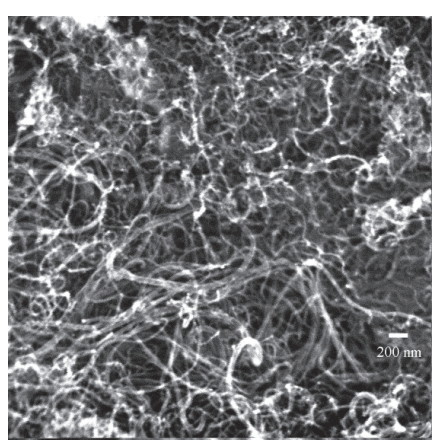

b

Рис. 1. СЭМ- изображения МУНТ

(a - MУHT-1 (Co-Mo/Al $\mathrm{O}_{3}-\mathrm{MgO}-$ катализатор), b - MУHT-2 (Fe-Co/ ${ }_{2.1} \mathrm{Al}_{2} \mathrm{O}_{3}-$ катализатор) 


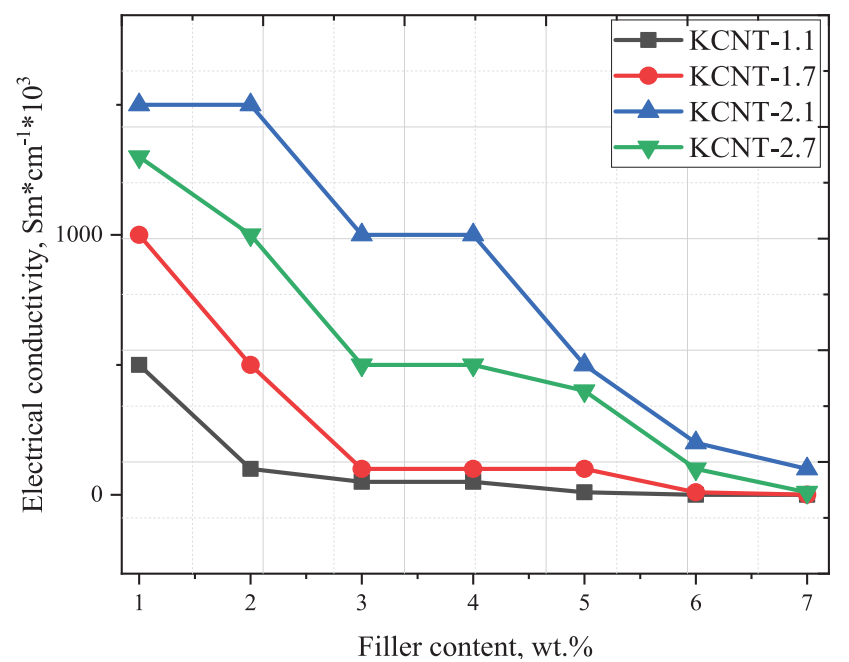

Рис. 2. Электропроводность нагревательных элементов на основе КУНТ-1.1, КУНТ-1.7, КУНТ-2.1 и КУНТ-2.7

На основе данных, представленных на рис. 3 следует, что для нагревательных элементов, изготовленных на основе КУНТ-1 с различной концентрацией, температурное поле, распределенное на поверхности образцов при различном содержании наполнителя (масс.\%) имеют равномерное распределение при разных температурных режимах. Также, следует отметить, что для всех нагревательных элементов, изменение концентрации МУНТ повлияло на значение верхнего порога рабочего электрического напряжения, которое составило 6-24 В, при этом, с ростом концентрации МУНТ данные значения напряжения увеличиваются.

\section{Результаты исследования композитов на взаимодействие с ЭМИ}

На рис. 4 представлена частотная зависимость коэффициентов прохождения ЭМИ для образцов композитных материалов на основе полидиметилсилоксана с различным содержанием масс.\% МУНТ-2 (рис. 4a и 4b). Для данного типа композит- ного материала наблюдается уменьшение значений коэффициентов пропускания с ростом концентрации добавки и уменьшение угла наклона к оси частот. Также, для образцов с концентрациями добавок 2 и 4 масс.\%, коэффициент пропускания остается постоянным в исследуемом диапазоне частот и составляет 30 и $20 \%$ соответственно. В диапазоне частот от 8 до 26 ГГц с увеличением концентрации от 1 до 2 масс.\% наблюдается рост коэффициента пропускания, а затем, с увеличением концентрации до 4 масс.\% происходит заметное уменьшение пропускания ЭМИ.

На рис. 4c и 4d представлены результаты исследования процессов взаимодействия электромагнитного излучения с композитными материаламина основе полидиметилсилоксана с различным добавлением масс.\% МУНТ-2. Образцы композитных материалов с МУНТ-2 демонстрируют большие значения коэффициентов отражения в сравнении с исходным полимером (рис. 4). Исследуемый параметр варьируется в пределах 40-50 \% для образцов с концентрациями добавки 1 и 2 масс.\%. Образец с концентрацией добавки в 4 масс.\% демонстрирует снижение значения коэффициента отражения от 60 до 40 \% в диапазоне частот 8-18 ГГц. В диапазоне от 18 до 40 ГГц, для данного образца наблюдается постоянное значение вблизи 45 \%.

В диапазоне частот от 26 до 40 ГГц, пропускание ЭМИ для образцов композитов данного типа остается постоянным и составляет порядка $15 \%$. В частотном диапазоне от 26 до 40 ГГц, образцы композитных материалов с данным типом добавки обладают наименьшим пропусканием ЭМИ. Что при сравнимых коэффициентах отражения приводит к большим значениям поглощенного ЭМИ образцами на основе МУНТ-1 (рис. 5).

\section{Заключение}

Исследованы тепловые эффекты в эластомерах с различной концентрацией МУНТ-1 и МУНТ-2 при различном уровне постоянного напряжения.

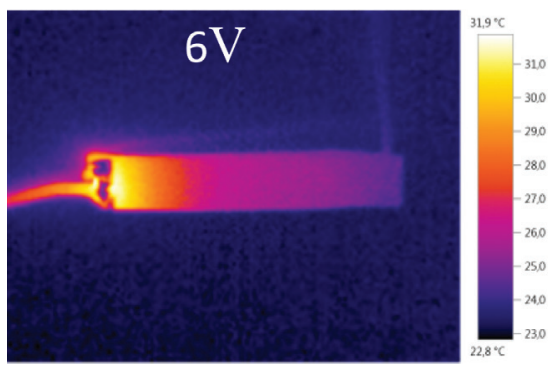

$\mathbf{a}$

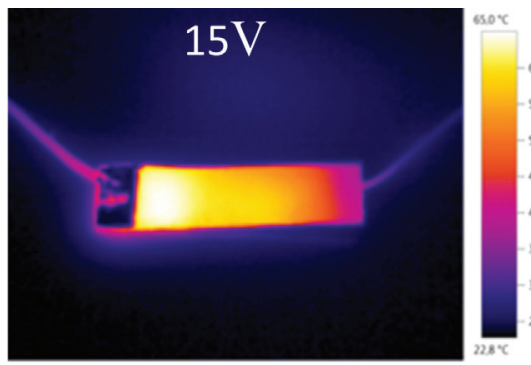

b

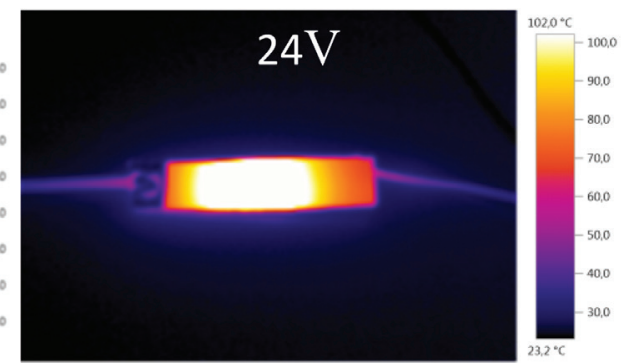

c

Рис. 3. Тепловизионные снимки нагревательных элементов, выполненные с помощью тепловизора Tеsto 875: $\mathbf{a}-K У H T-1.3, \mathbf{b}-K У H T-2.3, \mathbf{c}-K У H T-2.7$ 

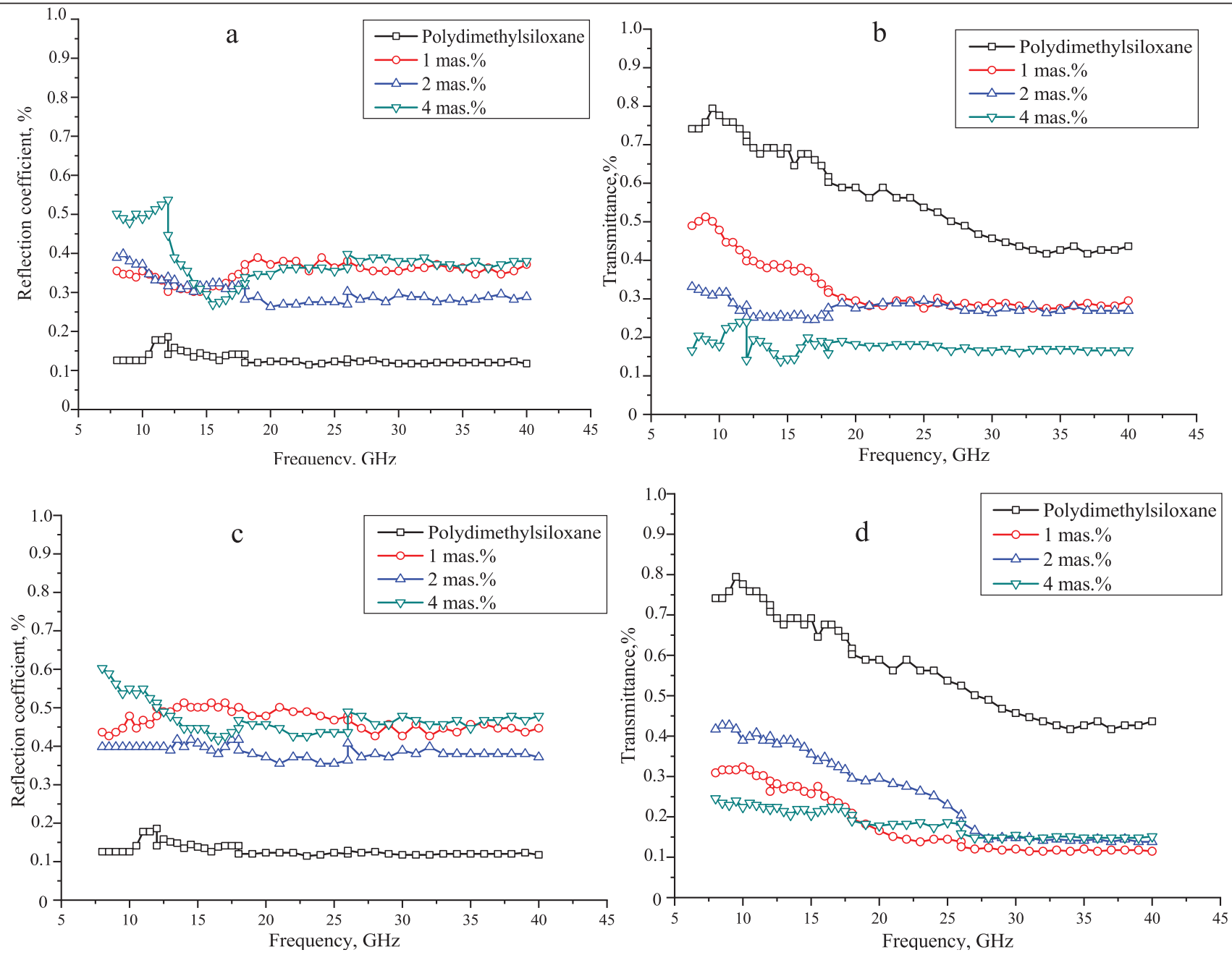

Рис. 4. Частотная зависимость коэффициентов отражения ЭМИ от композитных материалов с МУНТ-1 и МУНТ-2:

$\mathbf{a}$ - коэффициент отражения ЭМИ, образиов КУНТ-1.1 ...1.4, b - коэффициент пропускания ЭМИ, образиов КУНТ -1.1...1.4, с- коэффициент отражения ЭМИ, образцов КУНТ-2.1 ...2.4,

d - коэффициент пропускания ЭМИ, образцчов КУНТ-2.1 ..2.4
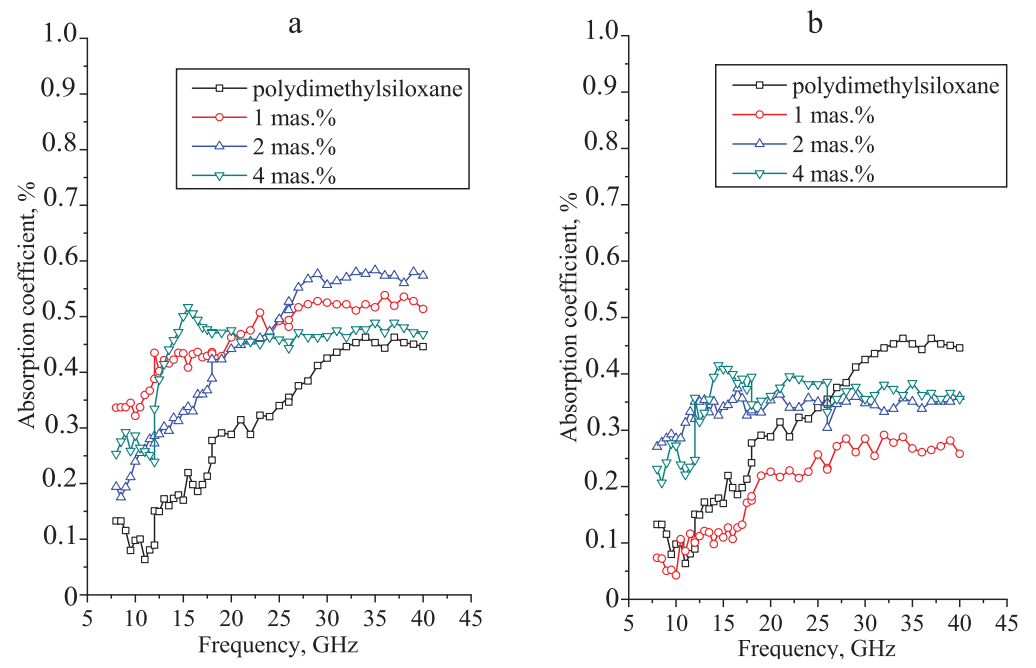

Рис. 5. Частотная зависимость коэффициентов поглощения ЭМИ композитными материалами:

$\mathbf{a}$ - коэффициент поглощения ЭМИ, образиов КУНТ-1.1...1.4,

$\mathbf{b}$ - коэффициент поглощеени ЭМИ, образиов КУНТ-2.1 ...2.4 
Максимальная температура нагрева нагревательных элементов, изготовленных из КУНТ-1 и КУНТ-2 составляла $102{ }^{\circ} \mathrm{C}$. По результатам проведенных исследований для процессов взаимодействия электромагнитного излучения с композитными материалами с добавлением МУНТ синтезированных на катализаторах $\mathrm{Co}-\mathrm{Mo} / \mathrm{Al}_{2} \mathrm{O}_{3}-\mathrm{MgO}$ и $\mathrm{Fe}-\mathrm{Co} / 2.1 \mathrm{Al}_{2} \mathrm{O}_{3}$ установлено, что 2-й тип наполнителя при максимальной концентрации наполнителя демонстрирует широкополосное ослабление (до $20 \%$ ) падающего излучения, в то же время, все образцы 1-го типа наполнителя проявляют способность к экранированию электромагнитного излучения в диапазоне от 26 до 40 ГГц на уровне $15 \%$. При сравнимых коэффициентах отражения данный тип наполнителя обладает и большей поглощающей способностью.

Использование в качестве полимерных матриц -полидиметилсилоксана обладающего высокой гибкостью, с добавками МУНТ полученных на различных катализаторах $\left(\mathrm{Co}-\mathrm{Mo} / \mathrm{Al}_{2} \mathrm{O}_{3}-\mathrm{MgO}\right.$ и $\mathrm{Fe}-\mathrm{Co} /{ }_{2.1} \mathrm{Al}_{2} \mathrm{O}_{3}$ ), позволяет сформировать гибкие нагревательные элементы с температурными режимами до $102{ }^{\circ} \mathrm{C}$ и выше, температура нагрева и рабочее напряжение таких нагревателей зависят от массового содержания МУНТ в объеме полимерной матрицы, с возможностью экранированию электромагнитного излучения в диапазоне от 26 до 40 ГГц на уровне $15 \%$.

Исследование выполнено при финансовой поддержке РФФИ в рамках научного проекта № 18-53-00032 Бел_а.

\section{Лuтература}

1. Lopera-Valle A., McDonald A. Journal of Thermal Spray Technology. 2015. V. 24. P. 1289-1301. DOI: 10.1007/s11666-015-0302-7.

2. Zhang W., Dehghani-Sanij A.A., Blackburn R.S. J. Mater. Sci. 2007. V. 42. P. 3408-3418. DOI: $10.1007 /$ s10853-007-1688-5.

3. Heaney M.B. Appl. Phys. Lett. 1996. V. 69. P. 2602 2604. DOI: $10.1063 / 1.117713$.

4. Markov A.V., Chizhov A.S. Fine Chemical Technologies. 2019. V. 14(2). P. 60-69. (in Russian) DOI: DOI: 10.32362/2410-6593-2019-14-2-60-69.

5. Goak J.C., Kim T.Y., Uk Kim D., Chang K.S., Lee C.S., Lee N. Applied Surface Science. 2020. V. 510. P. 145445. DOI: 10.1016/j.apsusc.2020.145445.
6. Azulay D., Eylon M., Eshkenazi O., Toker D., Balberg M.I. Phys. Rev. Lett. 2003. V. 90. N. 23. P. 236601. DOI: 10.1103/PhysRevLett.90.236601.

7. Tang H., Chen X., Tang A., Luo Y. J. Appl. Polym. Sci. 1996. V. 59. P. 383-387. DOI: $10.1002 /(\mathrm{SICI}) 1097-$ 4628(19960118)59:3<383::AID-APP1>3.0.CO;2-L.

8. Dafu W., Tiejun Z., Yi X.-S. J. Appl. Polym. Sci. 2000. V. 77. P. 53-58. DOI: $10.1002 /(\mathrm{SICI}) 1097-$ 4628(20000705)77:1<53::AID-APP8>3.0.CO;2-8.

9. Jang S.-H., Park Y.-L. Nanomaterials and Nanotechnology. 2018. V. 8(7). P. 1-8. DOI: $10.1177 / 1847980418776473$.

10. Yang L., Lia S., Zhou X., Liu J., Lia Y., Yang M., Yuan Q., Zhang W. Synthetic Metals. 2019. V. 253. P. 122130.

11. Bao S.P., Liang G.D., Tjong S.C. IEEE Transactions on Nanotechnology. 2009. V. 8(6). P. 729-736. DOI: 10.1109/TNANO.2009.2023650.

12. Rowlands W., Vaidhynathan B. Journal of the European Ceramic Society. 2019. V. 39(12). P. 3475-3483. DOI: 10.1016/j.jeurceramsoc.2019.03.024.

13. Lee J.H., Kim S.K., Kim N.H. Scripta Mater. 2006. V. 55(12). P. 1119-1122. DOI: 10.1016/j.scriptamat.2006.08.051.

14. Soares B.G., Calheiros L.F., Silva A.A., Indrusiak T., Barra G.M., Livi S. Journal of Applied Polymer Science. 2018. V. 135(24). P. 45564. DOI: 10.1002/app.45564.

15. Gao A., Zhao F., Wang F., Zhang G., Zhao S., Cui J. Composites Part A: Applied Science and Manufacturing. 2019. V. 122. P. 1-7. DOI: 10.1016/j.compositesa.2019.04.019.

16. Zhang W., Dehghani-Sanij A.A., Blackburn R.S. J. Mater Sci. 2007. V. 42. P. 3408-3418. DOI: $10.1007 /$ s10853-007-1688-5.

17. Jang S.-H., Kim D., Park Y.-L. Materials. 2018. V. 11(9). P. 1775. DOI: 10.3390/ma11091775.

18. Bao Su P., Liang G., Tjong S.C. IEEE Transactions on Nanotechnology. 2009. V. 8(6). P. 729-736. DOI: 10.1109/TNANO.2009.2023650.

19. Huang Y., Feng Y., Jiang T. Optics Express. 2007. V. 15(18). P. 11133-11141. DOI: 10.1364/ OE.15.011133.

20. Yong B.-Z., Cui T.-J. Progress In Electromagnetics Research B. 2011. V. 27. P. 151-163. DOI: 10.2528/ PIERB10081501.

21. Liu T., Zhou P.H., Xie J.L., Deng L.J. J. Appl. Phys. 2011. V. 110. P. 033918. DOI: $10.1063 / 1.3622144$.

22. Liang W.M., Jun Z.S., Qi L.J., Wei L., Mei L.X., Liang X.W. IEEE Proceeding, International Work Shop, Metamaterials (Meta). 1-4. Nanjing, Oct. 2012. DOI: 10.1109/META.2012.6464924.

23. De Volder M.A., Tawfick S.H., Baughman R.H., Hart A.J. Science. 2013. V. 339(6119). P. 535-539. DOI: $10.1126 /$ science. 1222453 . 